\title{
Theoretical reevaluations of the black hole mass - bulge mass relation - I. Effect of the seed black hole mass
}

\author{
Hikari Shirakata, ${ }^{1 \star}$ Toshihiro Kawaguchi, ${ }^{2}$ Takashi Okamoto, ${ }^{1}$ Ryu Makiya,,${ }^{3,4}$ \\ Tomoaki Ishiyama, ${ }^{5}$ Yoshiki Matsuoka,,${ }^{6,7}$ Masahiro Nagashima, ${ }^{8}$ Motohiro Enoki,${ }^{9}$ \\ Taira Oogi ${ }^{3}$ and Masakazu A. R. Kobayashi ${ }^{10}$ \\ ${ }^{1}$ Department of Cosmosciences, Hokkaido University, N10 W8, Kitaku, Sapporo, 060-0810, Japan \\ ${ }^{2}$ Department of Liberal Arts and Sciences, Sapporo Medical University, S1W17, Chuo-ku, Sapporo, 060-8556, Japan \\ ${ }^{3}$ Kavli Institute for the Physics and Mathematics of the universe, Todai Institutes for Advanced Study, the University of Tokyo, Kashiwa, 277-858 \\ ${ }^{4}$ Max-Planck-Institut fur Astrophysik, Karl-Schwarzschild Str. 1, D-85741 Garching, Germany \\ ${ }^{5}$ Institute of Management and Information Technologies, Chiba University, 1-33, Yayoi-cho, Inage-ku, Chiba, 263-8522, Japan \\ ${ }^{6}$ National Astronomical Observatory of Japan, Mitaka, Tokyo 181-8588, Japan \\ ${ }^{7}$ Department of Astronomy, School of Science, Graduate University for Advanced Studies, Mitaka, Tokyo 181-8588, Japan \\ ${ }^{8}$ Faculty of Education, Bunkyo University, Koshigaya, Saitama 343-8511, Japan \\ ${ }^{9}$ Faculty of Business Administration, Tokyo Keizai University, Kokubunji, Tokyo, 185-8502, Japan \\ ${ }^{10}$ Faculty of Natural Sciences, National Institute of Technology, Kure College, 2-2-11, Agaminami, Kure, Hiroshima, 737-8506, Japan
}

Accepted XXX. Received YYY; in original form ZZZ

\begin{abstract}
We explore the effect of varying the mass of the seed black hole on the resulting black hole mass - bulge mass relation at $z \sim 0$, using a semi-analytic model of galaxy formation combined with large cosmological $N$-body simulations. We constrain our model by requiring the observed properties of galaxies at $z \sim 0$ are reproduced. In keeping with previous semi-analytic models, we place a seed black hole immediately after a galaxy forms. When the mass of the seed is set at $10^{5} M_{\odot}$, we find that the model results become inconsistent with recent observational results of the black hole mass - bulge mass relation for dwarf galaxies. In particular, the model predicts that bulges with $\sim 10^{9} M_{\odot}$ harbour larger black holes than observed. On the other hand, when we employ seed black holes with $10^{3} M_{\odot}$, or randomly select their mass within a $10^{3-5} M_{\odot}$ range, the resulting relation is consistent with observation estimates, including the observed dispersion. We find that to obtain stronger constraints on the mass of seed black holes, observations of less massive bulges at $z \sim 0$ are a more powerful comparison than the relations at higher redshifts.
\end{abstract}

Key words: methods: numerical - galaxies: bulges - galaxies: nuclei - (galaxies:) quasars: supermassive black holes

\section{INTRODUCTION}

Observations have found luminous quasars at $z>6$, with masses of supermassive black holes (SMBHs) estimated at $\sim 10^{9} M_{\odot}$ (Mortlock et al. 2011; Wu et al. 2015). Larger SMBH masses at higher redshifts require either (i) relatively heavier seed black holes (BHs) with $\sim 10^{5} M_{\odot}$ (e.g., Lodato \& Natarajan 2006), or (ii) super-Eddington accretion for rapid growth of BHs (Rees 1992; Kawaguchi 2003). Both these mechanisms are potentially possible: (i) The massive seed BHs can be formed as the end products of gas collapse with virial temperatures $\geq 10^{4} \mathrm{~K}$ without molec-

* E-mail: shirakata@astro1.sci.hokudai.ac.jp ular cooling (Begelman et al. 2006). (ii) Estimations of accretion rates and duration of the super-Eddington accreting active galactic nuclei (AGNs), together with the observed trend of higher Eddington ratios at higher redshift (e.g., McLure \& Dunlop 2004; Nobuta et al. 2012), indicate that $\mathrm{BHs}$ have grown via super-Eddington accretion in early universe (Kawaguchi et al. 2004). These two mechanisms have been actively discussed.

Because of the uncertainty of the BH formation mechanism and limited computational resources, most cosmological hydrodynamic simulations and semi-analytic models of galaxy formation (hereafter SA models) have treated the formation of $\mathrm{BHs}$ by putting a seed $\mathrm{BH}$ with a set mass at the centre of each galaxy. For example, in Barber et al. (2016), who use the EAGLE simulation, a seed BH of 
$10^{5} h^{-1} M_{\odot}$ is placed by converting the bound gas particle with the highest density when a collapsed halo with $\geq 10^{10} h^{-1} M_{\odot}$ contains no BHs. Okamoto et al. (2008a), on the other hand, employ a seed $\mathrm{BH}$ mass with $10^{2} M_{\odot}$ and place the seed at the stellar density maxima when a virialized dark matter halo that consists of more than 1000 dark matter particles does not contain any BHs. Other cosmological simulations (e.g., Booth \& Schaye 2009) assume that the seed $\mathrm{BH}$ mass is proportional to the gas mass in the host halo. SA models treat the birth of BHs in mainly two different ways. In the first, a central BH is born when a galaxy initially merges with other galaxies (e.g., Kauffmann \& Haehnelt 2000; Enoki et al. 2003; Malbon et al. 2007; Lagos et al. 2009). In these models, the initial BH mass depends on the amount of the cold gas in the merging system. In the second method, a seed BH of a fixed mass is placed immediately after a galaxy forms: $10^{2} M_{\odot}$ (Menci et al. 2003), $10^{3} M_{\odot}$ (Fontanot et al. 2015), or $10^{5} M_{\odot}$ (Makiya et al. 2016). For a deeper understanding of seed BHs from SA models, Pezzulli et al. (2016) and Valiante et al. (2016) focus on BH growth only in early universe $(z \gtrsim 5)$ and suggest that $100 M_{\odot}$ seed BHs at $z \gtrsim 23$ accretes gas via major mergers at super-Eddington rates, forming $10^{5} M_{\odot}$ BHs at $z \sim 17$. The mass of the seed BHs has previously been presumed to have only a small impact on the statistical properties of galaxies, AGNs and SMBHs, unless the mass accretion rate depends on black hole mass and a large amount of gas from their host galaxies is accreted by the BHs.

Many observations (e.g., Kormendy \& Richstone 1995; Häring \& Rix 2004; McConnell \& Ma 2013; Magorrian et al. 1998) have suggested that the mass of SMBHs $\left(M_{\mathrm{BH}}\right)$ correlates with the stellar mass of their host bulges $\left(M_{\text {bulge }}\right)$ at $z \sim 0$ (hereafter the $M_{\mathrm{BH}}-M_{\text {bulge }}$ relation). They indicate a nearly proportional $M_{\mathrm{BH}}-$ $M_{\text {bulge }}$ relation. The $M_{\mathrm{BH}}-M_{\text {bulge }}$ relation may suggest that SMBHs have co-evolved with their host galaxies. This co-evolution of $\mathrm{BH}$ and host galaxies have motivated theoretical studies investigating the mechanism of $\mathrm{BH}$ feeding, AGN activities, and the energetic feedback from AGNs in the context of the galaxy formation (see, however, Jahnke \& Macciò 2011).

It is worth noting that recent observational studies (e.g., Graham 2012; Scott et al. 2013) have suggested that for $M_{\text {bulge }} \lesssim 10^{10} M_{\odot}$, the $M_{\mathrm{BH}}-M_{\text {bulge }}$ relation becomes quadratic instead of the linear relation found for more massive bulges. It has also suggested that this quadratic relation continues down to $M_{\mathrm{BH}} \sim 10^{5} M_{\odot}$.

In this Letter, we investigate whether the mass of seed BHs affects model predictions of the local $M_{\mathrm{BH}}-M_{\text {bulge }}$ relation at $M_{\text {bulge }} \lesssim 10^{10} M_{\odot}$ by using an SA model. In Section 2 we briefly review our models regarding the growth of SMBHs. In Section 3 we present our results. We summarize and discuss the results in Section 4.

\section{METHODS}

We employ a revised version of the SA model, "New $N u$ merical Galaxy Catalogue" ( $\nu^{2} \mathrm{GC}$; Makiya et al. 2016, hereafter M16), where the SMBH growth and AGN properties are summarized by Enoki et al. (2003), Enoki et al. (2014), and Shirakata et al. (2015). The revised model differs from M16 in the following points; (i) the model of merger driven spheroid formation and (ii) the growth of spheroids via disc instability. Further details are given in Sec. 2.1 Despite these changes, we will show later that the main result of this Letter remains unchanged even if we use the M16 model.

We create merging histories of dark matter haloes from state-of-the-art cosmological $N$-body simulations (Ishiyama et al. 2015), which have a high mass resolution and large volume compared to previous simulations (e.g., 4 times better mass resolution compared with Millennium simulations, Springel et al. 2005). Table 1 summarizes basic properties of the simulations used in this Letter. The $\nu^{2} \mathrm{GC}-\mathrm{L}$ simulation has $16^{3}$ times larger volume and the same mass resolution compared with the $\nu^{2} \mathrm{GC}$-SS simulation. When calculating the SMBH mass function at $z \sim 6$, we use the $\nu^{2} \mathrm{GC}-\mathrm{L}$ since $\mathrm{SMBHs}$ in the early universe are rare. The $\nu^{2} \mathrm{GC}-\mathrm{H} 2$ simulation has the same box size and $\sim 64$ times higher mass resolution compared with the $\nu^{2} \mathrm{GC}$-SS simulation. Since we are interested in small galaxies in this Letter, we employ the $\nu^{2} \mathrm{GC}-\mathrm{H} 2$ simulation in most cases, while the $\nu^{2} \mathrm{GC}$-SS simulation is used for resolution studies. The details of the merger trees are given in Ishiyama et al. (2015). We consider mergers of galaxies, atomic gas cooling, gas heating by UV feedback and feedbacks via supernovae and AGNs, and the growth of SMBHs by coalescence and gas fueling. More detailed descriptions are available in M16. Throughout this Letter, we assume a $\Lambda$ CDM universe with the following parameters: $\Omega_{0}=0.31$, $\lambda_{0}=0.69, \Omega_{\mathrm{b}}=0.048, \sigma_{8}=0.83, n_{\mathrm{s}}=0.96$, and a Hubble constant of $H_{0}=100 h \mathrm{~km} \mathrm{~s}^{-1} \mathrm{Mpc}^{-1}$, where $h=0.68$ (Planck Collaboration et al. 2014).

\subsection{Spheroid formation}

We assume that the spheroid within galaxies grows via starbursts and the migration of disc stars. In the M16 model, we considered starbursts only triggered in the major mergers of galaxies. Such a model, however, cannot reproduce AGN luminosity functions at low and high redshifts simultaneously (e.g., Enoki et al. 2014). In the revised version of the model in $\nu^{2} \mathrm{GC}$, we therefore assume that starbursts are triggered not only by major mergers of galaxies but also by minor mergers and disc instability.

For mergers, we introduce the model of merger drivenspheroid formation proposed by Hopkins et al. (2009) based on hydrodynamic simulations, similar to the SA model by Somerville et al. (2015). After dark matter haloes merge together, we regard the central galaxy in the most massive progenitor halo as the new central galaxy in the combined halo. We next consider a merger of two galaxies ${ }^{1}$ where the primary galaxy has a larger baryon mass (cold gas + stars) than a secondary galaxy. In all such merger events, we assume that all stars in the secondary are absorbed in the

1 We consider galaxy mergers via dynamical friction (centralsatellite mergers) and via random collisions (satellite-satellite merger) without following subhalo orbits. We use the merging rate by the random collision estimated by Makino \& Hut (1997). The detailed descriptions of these mergers are found in Sec. 2.5 in M16. 


\begin{tabular}{lllccc}
\hline Name & $N$ & $L\left[h^{-1} \mathrm{Mpc}\right]$ & $m\left[h^{-1} M_{\odot}\right]$ & $M_{\min }\left[h^{-1} M_{\odot}\right]$ & $M_{\max }\left[h^{-1} M_{\odot}\right]$ \\
\hline$\nu^{2}$ GC-L & $8192^{3}$ & 1120.0 & $2.20 \times 10^{8}$ & $8.79 \times 10^{9}$ & $4.11 \times 10^{15}$ \\
$\nu^{2}$ GC-SS & $512^{3}$ & 70.0 & $2.20 \times 10^{8}$ & $8.79 \times 10^{9}$ & $6.58 \times 10^{14}$ \\
$\nu^{2}$ GC-H2 & $2048^{3}$ & 70.0 & $3.44 \times 10^{6}$ & $1.37 \times 10^{8}$ & $4.00 \times 10^{14}$ \\
\hline
\end{tabular}

Table 1. Properties of the $\nu^{2} \mathrm{GC}$ simulations. $N$ is the number of simulated particles, $L$ is the comoving box size, $m$ is the individual mass of a dark matter particle, $M_{\min }$ is the mass of the smallest haloes $(=40 \times m)$ which corresponds to the mass resolution, and $M_{\max }$ is the mass of the largest halo in each simulation.

bulge of the primary, and a part of the disc (cold gas + stars) of the primary is also turned into the bulge component. We assume that cold gas that falls into the primary's bulge is consumed by a starburst. Similarly to M16, we assume that the starburst and the gas accretion onto the SMBH start at the same time. The beginning time is assigned randomly within the timestep.

We also introduce the spheroid formation by disc instability in order to form SMBHs at $z \sim 6$. The spheroids formed through this mechanism might become so-called 'pseudo bulges', although we do not differentiate bulges formed by this process from those formed by mergers. The inclusion of disc instabilities are important as strongly selfgravitating galactic discs are likely to be dynamically unstable. Moreover, without the disc instability, we cannot form high redshift $(z \sim 6)$ SMBHs with $M_{\mathrm{BH}} \gtrsim 10^{9} M_{\odot}$.

Following Mo et al. (1998) and Cole et al. (2000), a galactic disc becomes unstable when

$f_{\mathrm{DI}} \equiv \frac{V_{\mathrm{d}}}{\left(G M_{\mathrm{disc}} / r_{\mathrm{disc}}\right)^{1 / 2}}<f_{\mathrm{DI}, \mathrm{crit}}$

where $G$ is the gravitational constant, $V_{\mathrm{d}}, M_{\mathrm{disc}}$, and $r_{\mathrm{d}}$ are the rotation velocity, the mass of cold gas + stars, and the half mass radius of the galactic disc, respectively. Since $f_{\mathrm{DI} \text {,crit }}$ depends on the gas fraction and density profile of a galactic disc (e.g., Efstathiou et al. 1982; Christodoulou et al. 1995), we consider $f_{\mathrm{DI}, \mathrm{crit}}$ as an adjustable parameter, whose value is chosen so as to reproduce the observed stellar mass function of bulges at $z \sim 0$. We assume that an unstable disc is completely destroyed and reforms into a bulge with a starburst.

\subsection{Growth of SMBHs}

When galaxies merge with each other or the galactic disc becomes dynamically unstable, a starburst occurs and a small fraction of cold gas gets accreted by the SMBH via the following relation:

$M_{\mathrm{acc}}=f_{\mathrm{BH}} \Delta M_{*, \text { burst }}$,

where $M_{\text {acc }}$ is the accreted cold gas mass which is proportional to the stellar mass newly formed by a starburst,

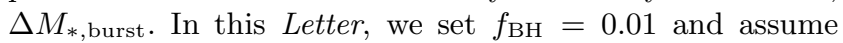
that the accretion rate onto an SMBH is not limited by the Eddington accretion rate.

SMBHs also grow when $\mathrm{BH}-\mathrm{BH}$ coalesce as their host galaxies merge. The timescale of the coalescence is difficult to determine. Similar to earlier work (e.g., Kauffmann \& Haehnelt 2000; Enoki et al. 2003; Somerville et al. 2008), we thus assume BHs merge instantaneously when their host galaxies merge.

\begin{tabular}{llllll}
\hline$\alpha_{\text {star }}$ & -2.131 & $\tau_{\mathrm{VO}}$ & $2.5 \times 10^{-9}$ & $\epsilon_{\mathrm{SMBH}}$ & 0.310 \\
$\epsilon_{\text {star }}$ & 0.203 & $f_{\mathrm{mrg}}$ & 1.0 & $\alpha_{\text {cool }}$ & 9.510 \\
$\alpha_{\text {hot }}$ & 4.080 & $\kappa_{\text {diss }}$ & 2.0 & $f_{\text {DI }, \text { crit }}$ & 1.1 \\
$V_{\text {hot }}$ & $129.7[\mathrm{~km} / \mathrm{s}]$ & $f_{\mathrm{BH}}$ & 0.01 & & \\
\hline
\end{tabular}

Table 2. Values of model parameters in this Letter.

We also consider radio-mode AGN feedback process as in Bower et al. (2006). Namely, when the halo cooling time becomes longer than the free-fall time at the cooling radius, and the cooling luminosity becomes smaller than the accretion luminosity, a hot halo gas is prevented from cooling.

\subsection{Seed Black Holes}

We place a seed $\mathrm{BH}$ immediately after a galaxy forms. In this Letter, we present results with $M_{\mathrm{BH} \text {,seed }}=10^{3} M_{\odot}$ (hereafter 'light seed model') where $M_{\mathrm{BH} \text {,seed }}$ is the seed $\mathrm{BH}$ mass, and $10^{5} M_{\odot}$ ('massive seed model'). We also test the case in which $M_{\mathrm{BH} \text {,seed }}$ takes uniformly random values in the logarithmic scale in the range of $3 \leq \log \left(M_{\mathrm{BH} \text {,seed }} / M_{\odot}\right) \leq 5$ (hereafter 'random seed model').

A galaxy is born when hot gas in the host halo cools efficiently. The hot gas cools by atomic cooling when the virial temperature, $T_{\text {vir }}$, is larger than $10^{4} \mathrm{~K}$. We also consider the heating effect by the UV background. We employ fitting formulae of the characteristic halo mass, $M_{c}(z)$, obtained from Okamoto et al. (2008b), below which haloes become baryon deficient. We find that runs $\nu^{2} \mathrm{GC}-\mathrm{SS}$ and -L do not resolve haloes with $M_{c}(z)$ in any redshift, while the $\nu^{2} \mathrm{GC}-\mathrm{H} 2$ does resolve haloes with $M_{c}(z)$ at $z \lesssim 5$ and those with $T_{\text {vir }}<10^{4}$ $\mathrm{K}$ at $z \lesssim 3$. This difference, however, does not affect the main conclusion of this Letter as we will show later.

\section{RESULTS}

In this section we present the effect of the seed BH mass on the $M_{\mathrm{BH}}-M_{\text {bulge }}$ relation at $z \sim 0$. Our model reproduces the observed galaxy luminosity functions, HI, BH, and bulge stellar mass functions at $z \sim 0$. Values of the model parameters used in this Letter are listed in Table 2. The description of the parameters is given in M16.

In Fig. 1, we present the $M_{\mathrm{BH}}-M_{\text {bulge }}$ relation at $z \sim 0$ predicted by the massive seed model (top panel) and light seed model (bottom panel). For the observational data with $M_{\mathrm{BH}} \lesssim 10^{6} M_{\odot}$, we use the data obtained from Graham \& Scott (2015) (hearafter GS15). This work reestimated the bulge and $\mathrm{BH}$ masses obtained by previous work (Jiang et al. 2011; Mathur et al. 2012; Scott et al. 
2013; Reines et al. 2013; Busch et al. 2014). We also plot LEDA 87300 whose $\mathrm{BH}$ mass is originally estimated by Baldassare et al. (2015) and re-evaluated by Graham et al. (2016). Almost all of the observational samples with $M_{\mathrm{BH}} \lesssim$ $10^{6} M_{\odot}$ have active BHs. In our model, the $M_{\mathrm{BH}}-M_{\mathrm{bulge}}$ relation does not change when we only plot AGNs.

Although all of our models reproduce the relation at $M_{\text {bulge }} \gtrsim 10^{10} M_{\odot}$, the massive seed model is inconsistent with the recent observational estimates for dwarf galaxies with $M_{\text {bulge }} \lesssim 10^{10} M_{\odot}$, in the sense that the predicted $\mathrm{BH}$ masses (shaded region) are larger than the observational estimates. We present the results by the $N$-body simulations with the same box size and different mass resolution $\left(\nu^{2} \mathrm{GC}-\right.$ SS and -H2) in all panels of Fig. 1. We find that the effect of the mass resolution of $N$-body simulations clearly appears with $M_{\text {bulge }} \lesssim 10^{9} M_{\odot}$. Nonetheless, the mass resolution does not affect our conclusion. Middle panel of Fig. 1 shows the result of the random seed model. We find that the random seed and light seed models reproduce the relation and its scatter well. These two successful models provide the same results in the range of $M_{\mathrm{BH}} \gtrsim 10^{5.5} M_{\odot}$ below which these models have significantly different slope of the relation. More observational data with $M_{\mathrm{BH}} \lesssim 10^{5.5} M_{\odot}$ are required for stronger constraints on the mass distribution of the seed BHs.

The sample of Jiang et al. (2011) seems to have the floor in the $M_{\mathrm{BH}}-M_{\text {bulge relation at }} M_{\mathrm{BH}} \sim 10^{5-6} M_{\odot}$. Jiang et al. (2011) thus support the results obtained from the simulations of Volonteri \& Natarajan (2009) with seed BHs of $10^{5} M_{\odot}$. On the other hand, our model prefers the lower seed $\mathrm{BH}$ mass than $10^{5} M_{\odot}$ to explain the $M_{\mathrm{BH}}-$ $M_{\text {bulge }}$ relation obtained from Jiang et al. (2011).

The black dashed and solid lines in Fig 1 depicts the scaling relations (Scott et al. 2013). ${ }^{4}$ Our models exhibit the slightly lower $M_{\mathrm{BH}}-M_{\text {bulge }}$ relation than the scaling relation at $M_{\text {bulge }} \gtrsim 10^{10} M_{\odot}$. In this region, $M_{\text {bulge }}$ evaluated from observations is potentially biased in favor of larger stellar masses (e.g., Shankar et al. 2016).

Our models also exhibit the transition of the slope in the $M_{\mathrm{BH}}-M_{\text {bulge }}$ relations from quadratic to near-linear. We also plot $M_{\mathrm{BH}}-M_{\text {bulge }}$ relations obtained by M16 in Fig. 1 , in which starbursts in the bulge and the gas fueling to a $\mathrm{BH}$ are only triggered by major mergers. They also show the "bend", meaning that bulge and $\mathrm{BH}$ growth via disc instability has small impact on the "bend" of the $M_{\mathrm{BH}}-M_{\mathrm{bulge}}$ relation. We have confirmed that stellar feedback is responsible for the quadratic relation as suggested by Fontanot et al. (2015).

Next, we investigate the origin of the scatter of the $M_{\mathrm{BH}}$ $-M_{\text {bulge }}$ relation. Fig. 2 indicates the distribution of the redshift at which galaxies newly form $\left(z_{\text {form }}\right)$. We predict that the scatter of the $M_{\mathrm{BH}}-M_{\text {bulge relation can be related to }}$ the difference of $z_{\text {form }}$. Three solid lines indicate the relation with different ranges of $z_{\text {form }}: z_{\text {form }}<4,4 \leq z_{\text {form }}<8$, and $8 \leq z_{\text {form }}$. We find that more massive systems form at

3 Originally obtained from Scott et al. (2013).

4 Scott et al. (2013) classified galaxies by their bulge surface brightness profiles: core-Sérsic galaxies (bulge surface brightness profiles have a partially depleted core) and Sérsic galaxies (bulge surface brightness profiles are well-fitted by a single Sérsic model).

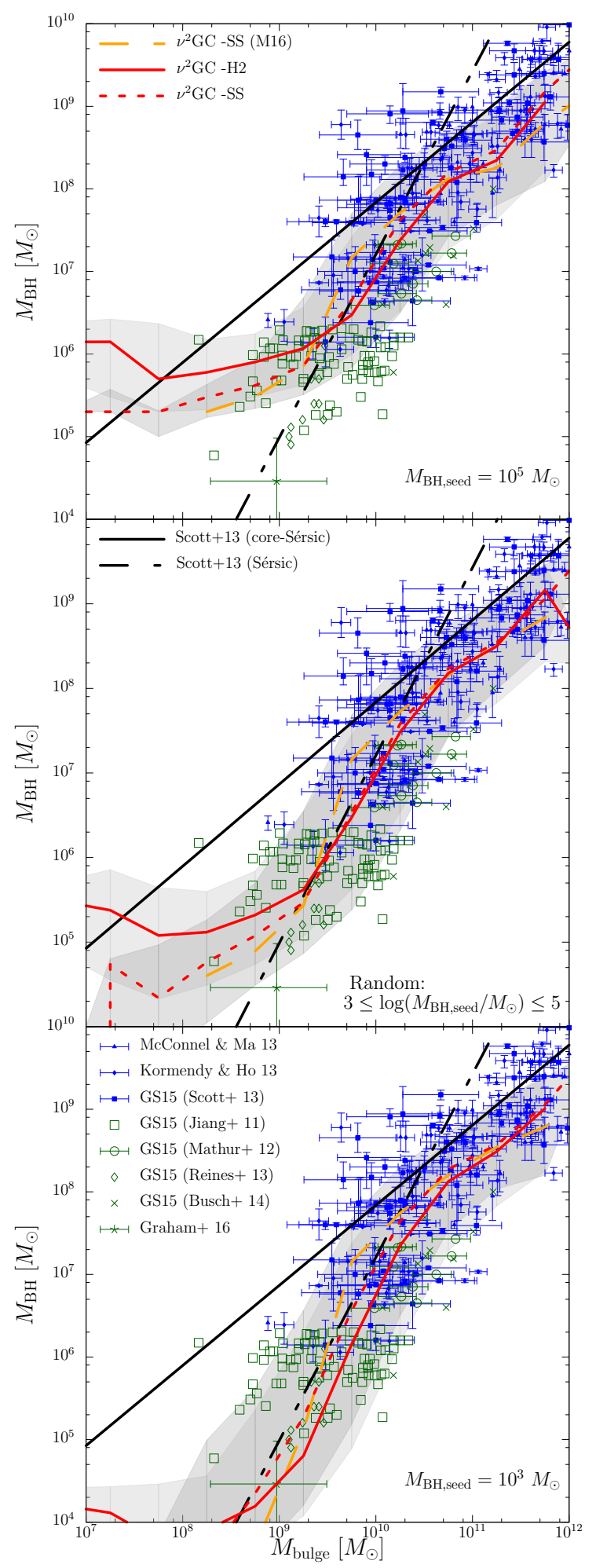

Figure 1. $M_{\mathrm{BH}}-M_{\text {bulge }}$ relations at $z \sim 0$ for different $M_{\mathrm{BH} \text {,seed }}$ the massive (top), random (middle), and light (bottom) seed models. Red dashed and solid lines present the results of the $\nu^{2} \mathrm{GC}-\mathrm{SS}$ and -H2 simulations, respectively. Red lines track the median, and shaded regions indicate 10-90 percentile of the models. Orange long dashed lines present the result of M16 with the $\nu^{2} \mathrm{GC}-\mathrm{SS}$ simulation. Blue filled symbols indicate observational results obtained from McConnell \& Ma (2013), Kormendy \& Ho (2013), and $\mathrm{GS}^{3} 5^{3}$ (triangles, diamonds, and squares, respectively). Green open symbols are AGN sample obtained from GS15,(see the text for more details). Green asterisks correspond LEDA 87300 (Graham et al. 2016; Baldassare et al.
2015). Black dot-dashed and solid lines depict the 1 scaling rela) tions (Scott et al. 2013). Since the $M_{\mathrm{BH}}-M_{\text {bulge }}$ relation is sensitive to the mass of seed $\mathrm{BHs}$, most seed $\mathrm{BHs}$ should not set to $10^{5} M_{\odot}$ for reproducing the observed local relation. 


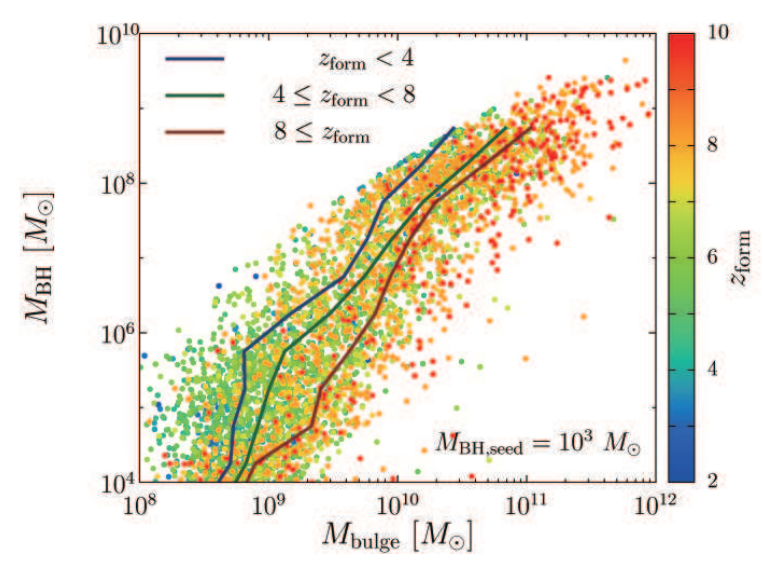

Figure 2. The $M_{\mathrm{BH}}-M_{\text {bulge }}$ relation at $z \sim 0$ with the $\nu^{2} \mathrm{GC}-$ SS simulation and $M_{\mathrm{BH} \text {,seed }}=10^{3} M_{\odot}$. The color indicates the redshift at which the host galaxies newly formed $\left(z_{\text {form }}\right)$. Solid lines shows the median value for different $z_{\text {form }}$ ranges; $z_{\text {form }}<4$ (dark brue), $4 \leq z_{\text {form }}<8$ (dark green), and $8 \leq z_{\text {form }}$ (dark red). For a given SMBH mass, bulges become more massive with higher $z_{\text {form }}$.

higher redshift. We also find that SMBHs become more massive with lower $z_{\text {form }}$ for a given $M_{\text {bulge }}$ and that the stellar mass of the bulge is larger with higher $z_{\text {form }}$ for a given $M_{\mathrm{BH}}$. These might be because large amount of gas gets accreted by the SMBHs immediately after $z_{\text {form }}$. Galaxies which formed later are subject to gas-rich processes, such as major mergers or disc instability. On the other hand, galaxies hosting the same mass of the SMBHs with higher $z_{\text {form }}$ have experienced more gas-poor processes of spheroid growth, such as dry mergers, than lower $z_{\text {form }}$ conterparts; their bulges increase their masses without feeding central BHs.

This trend is inconsistent with Merrifield et al. (2000) who suggest that $M_{\mathrm{BH}} / M_{\text {bulge }}$ ratios are higher in galaxies with older stellar age. We will investigate the origin of the scatter of $M_{\mathrm{BH}}-M_{\mathrm{bulge}}$ relation in a forthcoming paper.

\section{SUMMARY AND DISCUSSION}

We have investigated how the mass of the seed BHs affects model predictions of the local $M_{\mathrm{BH}}-M_{\text {bulge }}$ relation by using an SA model, $\nu^{2}$ GC. We find that seed BHs should not be dominated by those as massive as $10^{5} M_{\odot}$ to reproduce the observed $M_{\mathrm{BH}}-M_{\text {bulge }}$ relation at $z \sim 0$ over a wide range of bulge masses down to $M_{\text {bulge }} \lesssim 10^{10} M_{\odot}$. To obtain stronger constraints of the mass distribution for the seed BHs, observations of $M_{\mathrm{BH}} \lesssim 10^{5.5} M_{\odot}$ would be required.

The results in this Letter are consistent with cosmological hydrodynamic simulations performed by Anglés-Alcázar et al. (2015, 2016) which suggest that the $M_{\mathrm{BH}}-M_{\text {bulge }}$ relation converges independently of the seed $\mathrm{BH}$ mass at $M_{\text {bulge }} \gtrsim 10^{10} M_{\odot}$ while at $M_{\text {bulge }} \lesssim 10^{10} M_{\odot}$, seed BH mass becomes important in the scaling relation. Anglés-Alcázar et al. (2016) compare BH mass - galaxy stellar mass relations at $z \sim 0$ with $10^{4} h^{-1} M_{\odot}$ and $10^{6} h^{-1} M_{\odot}$ seed BHs. They find that in the case with $10^{6} h^{-1} M_{\odot}$ seed $\mathrm{BHs}$, the relation has a floor which also appears in the $M_{\mathrm{BH}}$

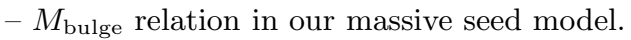

We explored whether the measurements of the $M_{\mathrm{BH}}-$

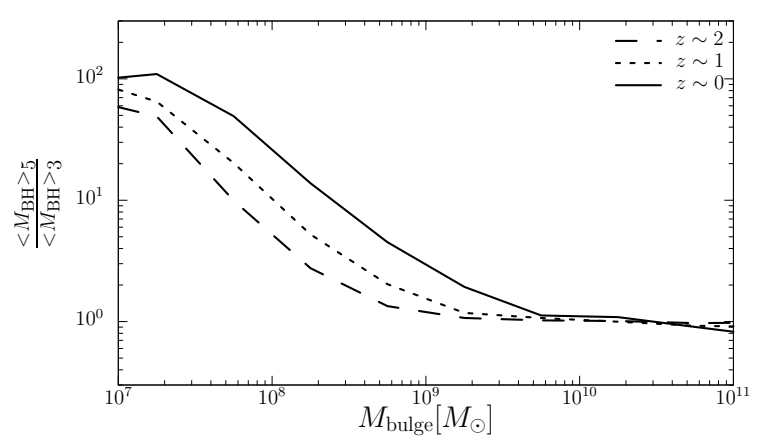

Figure 3. The difference of averaged SMBH mass due to the seed BH mass at $z \sim 2$ (dash-doted line), $z \sim 1$ (doted line), and $z \sim 0$ (solid line) as a function of their bulge stellar mass with the $\nu^{2} \mathrm{GC}-\mathrm{H} 2$ simulation. The difference becomes smaller at higher redshift.

$M_{\text {bulge }}$ relation at higher redshifts help to obtain further constraints on the mass of seed BHs. Fig. 3 depicts the ratio of the average $\mathrm{BH}$ masses in the light seed model $\left(\equiv\left\langle M_{\mathrm{BH}}\right\rangle_{3}\right)$ and those in the massive seed model $\left(\equiv\left\langle M_{\mathrm{BH}}\right\rangle_{5}\right)$, as a function of bulge masses obtained from the $\nu^{2} \mathrm{GC}-\mathrm{H} 2$ simulation. The difference in the seed mass clearly appears in galaxies with bulge mass below $3 \times 10^{9} M_{\odot}$ at $z \sim 0,1$ and 2 . We find that the difference due to the seed mass becomes smaller at higher redshift for a given $M_{\text {bulge }}$. Therefore observations of less massive bulges at $z \sim 0$ are more powerful than at higher redshifts for constraining the mass distribution of seed black holes.

Next we investigated SMBH mass functions at $z \sim 6$ by using the $\nu^{2} \mathrm{GC}-\mathrm{L}$ simulation. Fig. 4 shows the results of the light and massive seed models (blue and red circles, respectively). The SMBH mass function at $z \sim 6$ obtained from our model is nearly consistent with the estimation of Willott et al. (2010) in the range of $M_{\mathrm{BH}} \gtrsim 10^{7} M_{\odot}$. We find that an SMBH mass function at $z \sim 6$ in the range of $M_{\mathrm{BH}} \gtrsim 10^{5.8} M_{\odot}$ does not depend on the mass of the seed BHs due to the large amount of cold gas that gets accreted by the BHs. This is true even when we employ the M16 model, in which a major merger is the only trigger for a starburst and gas fueling to a BH. As mentioned in Sec. 2.1, a smaller number of SMBHs, however, form at $z \sim 6$ in the M16 model. Massive BHs have also grown efficiently by gas accretion triggered by the disc instability as suggested by Bower et al. (2006).

\section{ACKNOWLEDGEMENTS}

We appreciate the detailed review and useful suggestions by the anonymous referee, which have improved our paper. We also appreciate the English proofreading by E. J. Tasker, helpful comments by A. Graham and R. Valiante. We would like to thank C. Lacey for helpful comments for $\nu^{2}$ GC. T.K. was supported in part by an University Research Support Grant from the NAOJ. T.O. was financially supported by JSPS Grant-in-Aid for Young Scientists (B: 24740112). R.M. was supported in part by MEXT KAKENHI (15H05896). T.I. has been supported by MEXT HPCI STRATEGIC PROGRAM and MEXT/JSPS KAKENHI (15K12031) and 


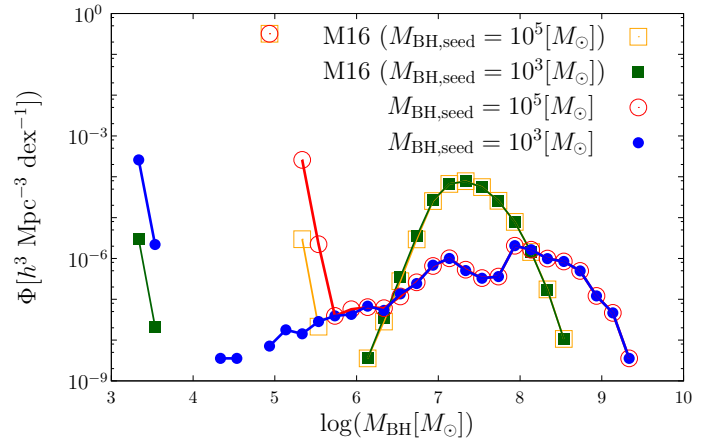

Figure 4. SMBH mass function at $z \sim 6$ based on the largest cosmological $N$-body simulation $\left(\nu^{2} \mathrm{GC}-\mathrm{L}\right)$. Orange open and green filled squares with lines show the results of M16, red open and blue filled circles with lines show the results of light and massive seed models, respectively. Even at $z \sim 6$, SMBH mass function does not depend on the mass of the seed $\mathrm{BHs}$ in the range of $M_{\mathrm{BH}} \gtrsim 10^{5.8} M_{\odot}$.

by Yamada Science Foundation. M.N. was supported by the Grant-in-Aid (25287049) from the MEXT of Japan.

\section{REFERENCES}

Anglés-Alcázar D., Özel F., Davé R., Katz N., Kollmeier J. A., Oppenheimer B. D., 2015, ApJ, 800, 127

Anglés-Alcázar D., Davé R., Faucher-Giguère C.-A., Özel F., Hopkins P. F., 2016, preprint, (arXiv:1603.08007)

Baldassare V. F., Reines A. E., Gallo E., Greene J. E., 2015, ApJ, 809, L14

Barber C., Schaye J., Bower R. G., Crain R. A., Schaller M., Theuns T., 2016, MNRAS,

Begelman M. C., Volonteri M., Rees M. J., 2006, MNRAS, 370,289

Booth C. M., Schaye J., 2009, MNRAS, 398, 53

Bower R. G., Benson A. J., Malbon R., Helly J. C., Frenk C. S., Baugh C. M., Cole S., Lacey C. G., 2006, MNRAS, 370, 645

Busch G., et al., 2014, A\&A, 561, A140

Christodoulou D. M., Shlosman I., Tohline J. E., 1995, ApJ, 443,551

Cole S., Lacey C. G., Baugh C. M., Frenk C. S., 2000, MNRAS, 319,168

Efstathiou G., Lake G., Negroponte J., 1982, MNRAS, 199, 1069

Enoki M., Nagashima M., Gouda N., 2003, PASJ, 55, 133

Enoki M., Ishiyama T., Kobayashi M. A. R., Nagashima M., 2014, ApJ, 794, 69

Fontanot F., Monaco P., Shankar F., 2015, MNRAS, 453, 4112

Graham A. W., 2012, ApJ, 746, 113

Graham A. W., Scott N., 2015, ApJ, 798, 54 (GS15)

Graham A. W., Ciambur B. C., Soria R., 2016, ApJ, 818, 172

Häring N., Rix H.-W., 2004, ApJ, 604, L89

Hopkins P. F., Cox T. J., Younger J. D., Hernquist L., 2009, ApJ, 691,1168

Ishiyama T., Enoki M., Kobayashi M. A. R., Makiya R., Nagashima M., Oogi T., 2015, PASJ, 67, 61

Jahnke K., Macciò A. V., 2011, ApJ, 734, 92

Jiang Y.-F., Greene J. E., Ho L. C., 2011, ApJ, 737, L45

Kauffmann G., Haehnelt M., 2000, MNRAS, 311, 576

Kawaguchi T., 2003, ApJ, 593, 69

Kawaguchi T., Aoki K., Ohta K., Collin S., 2004, A\&A, 420, L23

Kormendy J., Ho L. C., 2013, ARA\&A, 51, 511

Kormendy J., Richstone D., 1995, ARA\&A, 33, 581

Lagos C. D. P., Padilla N. D., Cora S. A., 2009, MNRAS, 395, 625
Lodato G., Natarajan P., 2006, MNRAS, 371, 1813

Magorrian J., et al., 1998, AJ, 115, 2285

Makino J., Hut P., 1997, ApJ, 481, 83

Makiya R., et al., 2016, PASJ, 68, 25 (M16)

Malbon R. K., Baugh C. M., Frenk C. S., Lacey C. G., 2007, MNRAS, 382, 1394

Mathur S., Fields D., Peterson B. M., Grupe D., 2012, ApJ, 754,146

McConnell N. J., Ma C.-P., 2013, ApJ, 764, 184

McLure R. J., Dunlop J. S., 2004, MNRAS, 352, 1390

Menci N., Cavaliere A., Fontana A., Giallongo E., Poli F., Vittorini V., 2003, ApJ, 587, L63

Merrifield M. R., Forbes D. A., Terlevich A. I., 2000, MNRAS, 313, L29

Mo H. J., Mao S., White S. D. M., 1998, MNRAS, 295, 319

Mortlock D. J., et al., 2011, Nature, 474, 616

Nobuta K., et al., 2012, ApJ, 761, 143

Okamoto T., Nemmen R. S., Bower R. G., 2008a, MNRAS, 385,161

Okamoto T., Gao L., Theuns T., 2008b, MNRAS, 390, 920

Pezzulli E., Valiante R., Schneider R., 2016, MNRAS, 458, 3047

Planck Collaboration et al., 2014, A\&A, 571, A1

Rees M. J., 1992, in Duschl W. J., Wagner S. J., eds, Physics of Active Galactic Nuclei. p. 662

Reines A. E., Greene J. E., Geha M., 2013, ApJ, 775, 116

Scott N., Graham A. W., Schombert J., 2013, ApJ, 768, 76

Shankar F., et al., 2016, MNRAS,

Shirakata H., Okamoto T., Enoki M., Nagashima M., Kobayashi M. A. R., Ishiyama T., Makiya R., 2015, MNRAS, 450, L6

Somerville R. S., Hopkins P. F., Cox T. J., Robertson B. E., Hernquist L., 2008, MNRAS, 391, 481

Somerville R. S., Popping G., Trager S. C., 2015, MNRAS, 453, 4337

Springel V., et al., 2005, Nature, 435, 629

Valiante R., Schneider R., Volonteri M., Omukai K., 2016, MNRAS,

Volonteri M., Natarajan P., 2009, MNRAS, 400, 1911

Willott C. J., et al., 2010, AJ, 140, 546

Wu X.-B., et al., 2015, Nature, 518, 512

This paper has been typeset from a $\mathrm{T}_{\mathrm{E}} \mathrm{X} / \mathrm{LAT} \mathrm{T} \mathrm{X}$ file prepared by the author. 